\title{
COMMENTARY
}

\section{Acute kidney injury: the dilemma of organ failures or just a circular reference?}

\author{
Anne-Sophie Guilbert and Pierre Tissières ${ }^{*}$ \\ See related research by Zwiers et al., http://ccforum.com/content/17/4/R151
}

\begin{abstract}
Whether the egg or the chicken came first remains debated, although the debate is not critical for those who love eating omelets. At least, it is well accepted that one needs to break eggs to fry an omelet. In some ways, there are similarities with acute kidney injury. A lot of debate arises from whether acute kidney injury is a complication of various insults and therapies or an expected continuum of the organ failure process. Indeed, it is well accepted that acute renal failure is associated with increased mortality in severely ill children and infants.
\end{abstract}

In this issue of Critical Care, Zwiers and colleagues [1] describe the incidence of acute kidney injury (AKI) in neonates on extracorporeal membrane oxygenation (ECMO) based on a 12-year cohort study from Sophia Children's Hospital Pediatric Intensive Care Unit in Rotterdam, The Netherlands. Of major interest, this study is looking at patients during an era when continuous renal replacement therapy (CRRT) was not systematically used, giving a clearer perspective on the natural kinetics of renal function, estimated by serum creatinine ( $\mathrm{SCr}$ ), during ECMO course. Interestingly, the worst renal function occurred early during the ECMO run, within the first 2 days, and with a 2- to 4-day delay until renal function improvement could be observed. This suggests that AKI is an early event in newborns requiring ECMO support. Establishing the 'natural history' of renal function in patients with multiple organ dysfunction syndrome (MODS) gives us the expected time period during which one may 'accept' to wait for spontaneous organ recovery. In this perspective, it would be valuable to know to what extent improving renal function during ECMO course really is associated with

\footnotetext{
* Correspondence: pierre.tissieres@bct.aphp.fr

Pediatric Intensive Care and Neonatal Medicine, Paris South University Hospitals, 78 Rue du Général Leclerc, 94275, Le Kremlin Bicetre, France
}

better outcome. Better characterization of MODS timing in the course of heterogeneous life-threatening diseases is central. International integrated database for the evaluation of severe sepsis and drotrecogin alfa (activated) therapy database analysis, regrouping 4,459 patients with sepsis, showed that worsening organ failures - particularly, cardiovascular, respiratory, renal, and hematologic failure - was the cause of death in patients with sepsis [2]. Interestingly, it showed that aggravating renal sequential organ failure assessment score was a late event close to death. One can hypothesize that bi-modal impairment in renal function should result in a more severe outcome. In children and neonates, similar data were published [3]. Aggravating pediatric logistic organ dysfunction score within the first 4 days was associated with a 50\% mortality. However, in newborns, renal dysfunction does not seem to have a major impact on mortality [4]. In the present study [1], the authors showed that severity of renal failure was strongly associated with mortality; the odds ratio of mortality for acute kidney failure (identified as an SCr of greater than $300 \%$ of normal value) was 12.7 . This suggests that, although knowing AKI natural course during ECMO may give arguments for delaying CRRT, there is clearly a cutoff value at which AKI severity directly impacts mortality. It is not the purpose of this commentary to discuss the ideal timing of CRRT during ECMO, because many questions remain to be debated, but this study confirms that AKI is a major outcome determinant in the course of ECMO.

If not an unresolved dilemma, AKI in the course of MODS remains a circular reference: AKI in patients requiring ECMO therapy is triggered by initial systemic insults, already present at the time of ECMO initiation, and aggravated by reperfusion injury and ECMO circuitinduced inflammation. The close interplay between the cause and severity of the disease, supportive therapies, and host response is influencing the development of AKI and MODS. Identifying as well as determining the relative importance of extrinsic factors a ggravating MODS/AKI 
during ECMO therapy is central. Besides technologic advances in ECMO circuit biocompatibility improvement, a lot of work is needed in monitoring organ 'reperfusion' as well as function, identifying deleterious therapeutic interventions, and improving standard therapies, such as antimicrobial chemotherapy. AKI during ECMO is not a dead-end complication. Recently, it was shown in an autopsy series of patients dying of septic shock that renal dysfunction was due mostly to focal renal tubular injury through apoptosis and not autophagy [5]. That study clearly suggested that renal dysfunction found in patients dying of sepsis-induced MODS is reversible. All organsupport therapies are now central in managing critically ill children. ECMO is certainly among the greatest recent advances, making a surgical theater technique widely available in the critical care setting for clinical conditions far away from the original cardiovascular indications. However, a lot remains to be done in order to identify proper indications, optimize all organ support, reduce complications, and better characterize pathophysiologic and cellular mechanisms of organ dysfunctions in children under ECMO.

\section{Abbreviations}

AKI: acute kidney injury; CRRT: continuous renal replacement therapy; ECMO: extracorporeal membrane oxygenation; MODS: multiple organ dysfunction syndrome; SCr: serum creatinine.

\section{Competing interests}

The authors declare that they have no competing interests.

Published: 04 Sep 2013

\section{References}

1. Zwiers AJ, de Wildt SN, Hop WC, Dorresteijn EM, Gischler SJ, Tibboel D, Cransberg $\mathrm{K}$ : Acute kidney injury is a frequent complication in critically ill neonates on extracorporeal membrane oxygenation: a 14-year cohort study. Crit Care 2013, 17:R151.

2. Vincent $J \mathrm{~L}$, Nelson $\mathrm{DR}$, Williams MD: Is worsening multiple organ failure the cause of death in patients with severe sepsis? Crit Care Med 2011, 39:1050-1055.

3. Leteurtre S, Duhamel A, Grandbastien B, Proulx F, Cotting J, Gottesman R, Joffe A, Wagner B, Hubert P, Martinot A, Lacroix J, Leclerc F: Daily estimation of the severity of multiple organ dysfunction syndrome in critically ill children. CMAJ 2010, 182:1181-1187.

4. Bestati N, Leteurtre S, Duhamel A, Proulx F, Grandbastien B, Lacroix J, Leclerc F: Differences in organ dysfunctions between neonates and older children: a prospective, observational, multicenter study. Crit Care 2010, 14:R202.

5. Takasu O, Gaut JP, Watanabe E, To K, Fagley RE, Sato B, Jarman S, Efimov IR, Janks DL, Srivastava A, Bhayani SB, Drewry A, Swanson PE, Hotchkiss RS: Mechanisms of cardiac and renal dysfunction in patients dying of sepsis. Am J Respir Crit Care Med 2013, 187:509-517.

$10.1186 / \mathrm{cc} 12873$

Cite this article as: Guilbert and Tissières: Acute kidney injury: the dilemma of organ failures or just a circular reference? Critical Care 2013, 17:182 